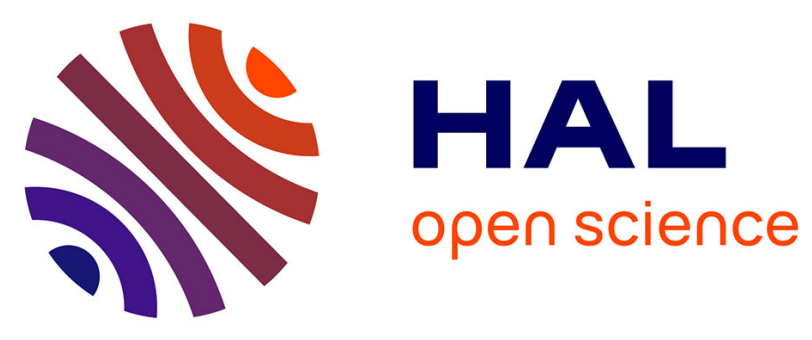

\title{
A novel microstructural interpretation for the biomechanics of mouse skin derived from multiscale characterization
}

Barbara Lynch, Stéphane Bancelin, Christelle Bonod-Bidaud, Jean-Baptiste Gueusquin, Florence Ruggiero, Marie-Claire Schanne-Klein, Jean-Marc Allain

\section{To cite this version:}

Barbara Lynch, Stéphane Bancelin, Christelle Bonod-Bidaud, Jean-Baptiste Gueusquin, Florence Ruggiero, et al.. A novel microstructural interpretation for the biomechanics of mouse skin derived from multiscale characterization. Acta Biomaterialia, 2017, 50, pp.302-311. 10.1016/j.actbio.2016.12.051 . hal-01531321

\section{HAL Id: hal-01531321 \\ https://hal.science/hal-01531321}

Submitted on 1 Jun 2017

HAL is a multi-disciplinary open access archive for the deposit and dissemination of scientific research documents, whether they are published or not. The documents may come from teaching and research institutions in France or abroad, or from public or private research centers.
L'archive ouverte pluridisciplinaire HAL, est destinée au dépôt et à la diffusion de documents scientifiques de niveau recherche, publiés ou non, émanant des établissements d'enseignement et de recherche français ou étrangers, des laboratoires publics ou privés.

$$
\text { Copyright }
$$


A novel microstructural interpretation for the biomechanics of mouse skin derived from multiscale characterization

Barbara Lynch ${ }^{1}$, Stéphane Bancelin ${ }^{2}$, Christelle Bonod-Bidaud ${ }^{3}$, Jean-Baptiste Gueusquin ${ }^{1}$, Florence Ruggiero $^{3}$, Marie-Claire Schanne-Klein ${ }^{2}$, Jean-Marc Allain ${ }^{1,4}$

(1) LMS, Ecole Polytechnique, CNRS, Université Paris-Saclay, Palaiseau, France

(2) LOB, Ecole Polytechnique, CNRS, INSERM, Université Paris-Saclay, Palaiseau, France

(3) Institut de Génomique Fonctionnelle de Lyon, ENS-Lyon, CNRS, Université de Lyon, Lyon, France

(4) Inria, Université Paris-Saclay, Palaiseau, France

Corresponding author:

Jean-Marc ALLAIN

allain@Ims.polytechnique.fr

Tel:+33-1-72925940; Fax:+33-1 69333026

Postal address: LMS, Ecole Polytechnique, 91128 Palaiseau cedex, France 


\section{Abstract}

Skin is a complex, multi-layered organ, with important functions in the protection of the body. The dermis provides structural support to the epidermal barrier, and thus has attracted a large number of mechanical studies. As the dermis is made of a mixture of stiff fibres embedded in a soft non-fibrillar matrix, it is classically considered that its mechanical response is based on an initial alignment of the fibres, followed by the stretching of the aligned fibres. Using a recently developed setup combining multiphoton microscopy with mechanical assay, we imaged the fibres network evolution during dermis stretching. These observations, combined with a wide set of mechanical tests, allowed us to challenge the classical microstructural interpretation of the mechanical properties of the dermis: we observed a continuous alignment of the collagen fibres along the stretching. All our results can be explained if each fibre contributes by a given stress to the global response. This plastic response is likely due to inner sliding inside each fibre. The non-linear mechanical response is due to structural effects of the fibres network in interaction with the surrounding non-linear matrix. This multiscale interpretation explains our results on genetically-modified mice with a simple alteration of the dermis microstructure. 


\section{Introduction}

The skin is a complex organ, with major physiological functions as regulation of the body temperature and hydration, and protection against physical aggressions and penetration of external agents. It is a multi-layered organ: starting from the outer surface, the skin comprises first the epidermis (a barrier made of epithelial cells), the dermis (a connective tissue that supports the epidermis) and finally the hypodermis (an adipose tissue). It is classically assumed that the dermis provides the main mechanical properties in stretching [1-3], although the other layers may be important for other loadings [4-6].

The dermis is a collagen-rich tissue, mostly composed of extracellular matrix with few cells inside. The matrix is a water solution of biomolecules, including proteoglycans, glycoproteins, and collagens, by far the most abundant protein. The collagen self-organizes into fibrils that further align into fibres in skin dermis. Dermal fibrils are heterotypic fibrils composed mainly of type I collagen, with other collagen types (as collagen $\mathrm{V}$ ) as minor components. The dermis can then be viewed as a network of fibres (collagen as well as elastin) surrounded by an unorganized non-fibrillar matrix.

The macroscopic mechanical properties of the dermis are well-known $[7,8]$. They are similar to the ones of other collagen-rich tissues, as tendon [9], cornea [10] or aorta [11]. First, they support large deformations (typically $50 \%$ for the skin, which is much larger than in other tissues) with a classical response in three parts. At low stretch, there is the toe-region, in which almost no force is recorded. Then, the force increases non-linearly in the heel-region, before reaching a linear part. At the end of this part, the tissue breaks. On top of this non-linear behaviour, the tissue exhibits viscous effects, not surprising for such hydrated material, with a modification of the force as a function of the loading rate, but also hysteresis loops if unloaded [12]. It may be noticed that repeated cyclic loadings lead also to a progressive shift of the response toward a limit cycle: it is the preconditioning effect $[3,13,14]$, which can be compared to the Mullin's effect in elastomers.

The relationship between these complex properties and the microstructure of the tissue has attracted a lot of theoretical attention, leading to the so-called "microstructural" models 
$[2,13,15,16]$. Indeed, being able to predict the effects of an alteration of the microstructure on the macroscopic properties is of first interest for biological and medical applications. As examples, microstructural models have been used to predict the effects of surgical treatments [17] and the consequences of pathological or age-related alterations of the collagen [18].

The mechanical interpretation behind all these models is an alignment of the collagen fibres in the toe and heel regions, followed by the stretching of the fibres in the linear part $[7,19,20]$. However, only few papers have investigated experimentally this interpretation in skin, due to the difficulty to image the evolution of collagenous microstructure in this complex, disorganized medium. First qualitative observations were done with SEM $[19,20]$, using a new sample for each image. It is only recently that it has become possible to observe the collagenous microstructure on the same sample at all stretch levels [21]. This was permitted by the development of mechanical assays combined with multiphoton microscopy $[9,22]$, and in particular Second Harmonic Generation (SHG) microscopy. Thereby, we can now get three-dimensional images specific of the fibrillary collagen, without staining [23-26]. This approach has been used very recently on skin [21,27], but also on other tissues as aorta [22,28], cornea [10], foetal membrane [29,30] or liver capsule [31]. These observations have been challenging the classical interpretation of the link between microstructure and mechanical properties.

In this paper, we present a series of experimental results, probing the different aspects of the mechanical response of skin (uniaxial stretching, cyclic loadings, relaxation...), but also the reorganization of the microstructure during a stretching assay. We propose a new interpretation to explain all our observations in a single frame. We use this new interpretation in order to explain the consequences of an alteration of the collagen synthesis in genetically-modified mice.

\section{Material and methods}

\subsection{Skin samples}


The experiments were performed on ex vivo skin from one-month-old control (Wild Type, WT) mice ( $n=25$ under the multiphoton microscope and $n=7$ outside) and from transgenic mice with a modified collagen $V$ synthesis ( $n=9$ under the multiphoton microscope and $n=7$ outside) from the same litters (referred to as K14-COL5A1).

All animal experiments were performed under animal care procedures and conducted in accordance with the guidelines set by the European Community Council Directives (86/609/EEC). All experimental procedures were approved by the Direction of the Veterinary Service of Rhone Department (DDSV, Lyon, France).

The transgenic mouse line was described in details elsewhere [32]. Briefly, it overexpresses the human proa1(V) chain in the epidermis, leading to the deposition of collagen $\mathrm{V}$ homotypic fibrils in the dermis. These small fibrils have a diameter smaller than $10 \mathrm{~nm}$, as observed on very young mice (5 days old) [32]. No other significant change in the dermis microstructure was reported on these very young mice.

Skin samples from the back of mice were depilated and de-epidermilized. The right foreleg was spotted with black ink to identify the head-tail orientation. They were stored in suitable culture medium at $4^{\circ}$ and used within 5 days for experiments as previously described [21].

\subsection{Experimental set-up}

Complementary sets of mechanical assays were performed on skin samples: (i) tensile tests, (ii) cycling experiments, and (iii) relaxation experiments. Some tests were coupled with multiphoton microscopy observations in order to provide multiscale biomechanical data, and some other ones were performed outside the multiphoton microscope, in a simpler set-up, for practical reasons.

The complete protocol of multiscale tensile tests has been described in a previous paper [21]. Briefly, skin samples were cut into a dog-bone shape and attached to the traction device, with a motor and a force sensor on each side. Firm gripping was obtained by pinching the skin between two metallic plates, with a small rubber joint (diameter $1 \mathrm{~mm}$ ) protruding partly above the lower plate. The skin 
samples dried and became very stiff between the joint and the upper plate, ensuring good fixation. We considered the distance between the two joints as the initial length.

The main direction of traction was the anatomical head-tail direction. The traction device was inserted in place of the microscope stage in the multiphoton microscope, with papillary dermis side facing the objective lens. Immersion gel (Lacrygel, Europhta) was used to ensure optical contact with the lens and prevent skin dehydration. A schematic representation of the experimental set-up under the multiphoton microscope is shown in Fig. 1a.

The tensile tests were performed with an incremental loading with steps of 0.05 strain, at a slow strain rate $\left(10^{-4} \mathrm{~s}^{-1}\right)$, until rupture of the sample. This strain rate was chosen so that we could follow the same region of the sample throughout the test. Indeed, although the traction device was symmetric, we had to slightly adjust the lateral and axial positions of the whole traction device under the multiphoton microscope by means of micrometre screws. A z-stack of multiphoton images was recorded at each step, over $50 \mu \mathrm{m}$ with an axial step of $2 \mu \mathrm{m}$, while the motors were stopped to avoid skin movements (see Fig. 2). The multiphoton imaging usually took around 5 minutes, during which the skin was allowed to relax freely. The relaxation did not seem to impact the fibres organization, as shown by recording subsequent z-stacks at the same imposed stretch (data not shown). The analysis of these relaxations will be described thereafter.

The force was recorded every second. The mechanical macroscopic response was obtained from the force $(F)$ and the initial sample section $\left(S_{0}\right)$ by calculating the nominal stress $\left(F / S_{0}\right)$. The imposed stretch ratio was quantified using the global stretch ratio $\lambda$ between the jaws, which was found to be equal to the average stretch in the field of view of the multiphoton microscope (around $300 \times 300$ $\mu \mathrm{m}^{2}$ ), determined by tracking the displacements of the centres of the hair follicles [21]. The nominal stress/imposed stretch curve (denoted stress/stretch curve hereafter for simplicity) obtained was a Jshaped curve, as expected from the literature, with a toe region (practically no stress is recorded), a heel region (stress builds up), then a linear part followed by a force plateau before sample rupture 
(see Fig. 2). We quantified the slope of the linear part, called the tangent modulus, and the heel region length of the stress/stretch curve as previously described [21].

We used the multiphoton microscope to acquire Second Harmonic Generation (SHG) images that specifically reveal collagen fibres without any labelling [23-25,33,34] (see Fig. 1a). Images stacks were epi-collected using a 20x, 0.9 NA objective lens (Olympus) with typically $30 \mathrm{~mW}$ laser power at focus as previously described [21]. We analysed these SHG images to obtain information on the evolution of skin's microstructure during the mechanical test, namely the evolution of the fibres network organization. The fibre orientation in every pixel was obtained using morphological filtering by a rotating linear structuring element. In brief, each image was filtered by a mean filter ( 3 pixels radius), and a morphological opening was applied using a 21 pixel $(9 \mu \mathrm{m})$ linear element at each point. The linear element was then rotated, giving a value of the opening for each angle; the orientation of the maximum value of the opening was then considered as the fibre orientation at this point of the image (for a more complete description, see Supplementary Methods in [21]). A normalized histogram of the fibre orientation distribution was calculated for each deformation step by merging the histograms of the more intense SHG images over a thickness of $10 \mu \mathrm{m}$ (6 images every $2 \mu \mathrm{m})$. A quantitative parameter, the orientation index (OI), was computed as previously described [21], and can be interpreted as the fraction of fibres aligned in the main orientation. It is maximal (100\%) for perfectly aligned fibres and zero for perfectly disorganized tissues.

Other mechanical tests were carried out outside the multiphoton microscope. In that case, the sample surfaces were labelled with a pattern of dots of Indian ink using a soft brush before being attached to the traction device. They were otherwise handled exactly as previously described. The samples were lit with white light using a LED light (Schott, KL 2500 LED), and images were recorded every second during the test using a digital camera (Pike, Allied Vision Technologies, with objective Tamron SP AF 90mm F/2.8 Di Macro 1:1). The pattern of Indian ink dots created the macroscopic pattern needed to analyse the experiments with Digital Image Correlation in post treatment using CorrelManuV software. This allowed us to measure the strain, which was found to be homogeneous 
at the millimetre scale. In the experiments outside the microscope, the hydration of the sample was ensured by spraying mineral water on the samples every other minute. A schematic representation of the experimental set-up outside the multiphoton microscope and of the labelled skin is shown in Fig. $1 b$.

The tensile tests were performed at a strain rate of $10^{-4} \mathrm{~s}^{-1}$, without any stops, until rupture of the sample. Details on the relaxation and cyclic experiments are presented in the following sections.

\subsection{Relaxation analysis}

Short relaxations: In the experiments combining mechanics and multiphoton microscopic observations, the traction had to be stopped every 0.05 strain to image the sample while it was immobile. While we maintained the global deformation constant, the stress relaxed because of skin's viscoelasticity. The incremental loading protocol lets us access relaxations from the same sample, at different stress levels.

To fit the relaxation curves, we used a series of exponentials with decreasing magnitude, called Prony Series, as suggested by Fung for connective tissues [11]. A one exponential function was found to be not sufficient to fit our data. We fitted the relaxation curves on the force/time curve with a biexponential function with saturation:

$$
F(t)=F_{1} \cdot e^{-t / t_{1}}+F_{2} \cdot e^{-t / t_{2}}+F_{3} .
$$

The fitting procedure was done using a Matlab script (The MathWorks, Natick, USA), using a Levenberg-Marquardt algorithm. Initial parameters were chosen as: $F_{1}=1, F_{2}=1, F_{3}=1, t_{1}=10$ s and $t_{2}=100 \mathrm{~s}$. Other initial values were tested when the fit residual was beyond the experimental noise; these new initial values were chosen based on the values from previous fits. A bi-exponential fit of a 6-minute long relaxation, recorded during multiphoton imaging, is shown in Fig. 3a.

$F_{1}, F_{2}$ and $F_{3}$ were divided by the initial force before relaxation, $F(0)$, to have relative proportions of force relaxed. The relaxation analysis provided eventually five parameters: the relative amplitude for short time relaxation $a_{1}\left(a_{1}=F_{1} / F(0)\right)$, the relative amplitude for long time relaxation $a_{2}\left(a_{2}=F_{2} / F(0)\right)$, 
the relative constant for the relaxation $s_{0}\left(s_{0}=F_{3} / F(0)\right)$ and two relaxations times, $t_{1}$ (short time) and $t_{2}$ (long time). Notably, the three amplitudes are interdependent: $a_{1}+a_{2}+s_{0}=1$.

The relaxations were sorted out manually in two categories, depending on whether they belonged to the heel region or the linear part of the stress/stretch curve. The identification of the relaxations in the toe region was impossible due to too low signal-to-noise ratio. A total of 43 relaxations were fitted for WT samples in the heel region, and 136 in the linear part, while 22 heel region and 49 linear part relaxations were analysed for the transgenic mice.

Long relaxations: Four longer relaxation experiments were also performed outside the multiphoton microscope. The sample was stretched up to a stretch ratio of 1.3 with a strain rate of $10^{-3} \mathrm{~s}^{-1}$. The motors were then stopped and the sample was allowed to relax freely for 3 to 8 hours. For those longer relaxations, more than two exponentials could be used to fit the force/time curve. However, the relative amplitudes for the smaller times were very small (less than $5 \%$ ), and thus difficult to identify precisely. A bi-exponential fit is thus the most suitable for long time relaxations, although different time scales are probed than for shorter time relaxations. A bi-exponential fit of a 6-hour long relaxation can be seen in Fig. $3 \mathrm{~b}$.

\subsection{Cycling experiments}

Ten tensile tests with a cyclic loading path were performed on WT samples without multiphoton observation. No cyclic experiments were carried out on genetically-modified mice skin due to low availability of the samples.

We performed two complete cycles with a maximum imposed stretch ratio of $\lambda=1.05$, then two cycles at $\lambda=1.1$, two cycles at $\lambda=1.2$, two cycles at $\lambda=1.3$, and then finally stretched the sample up to rupture (see Fig. 4a). The strain rate was $10^{-3} \mathrm{~s}^{-1}$.

We observed that the free length of the sample increased between the first and second cycle at the same imposed stretch ratio. To quantify this effect, we measured for each loading part of a cycle the stretch ratio $\lambda_{0}$ for which the force first exceeded the sensor's noise level (0.02 N).

\subsection{Statistics}


All skin samples were included in the statistical analysis, unless experimental data were not available for well identified reasons. For the fitting of relaxations curves, the data were not included when the signal-to-noise ratio prevented a proper fit (toe region of the stress/stretch curve), as well as when the relaxation curve was affected by the beginning of sample failure, when nearing the end of the experiment.

Error bars correspond to Standard Error of the Mean. Statistical tests were performed with $R(R$ development core team, $R$ foundation for statistical computing). We verified both the normality of the distribution and the equality of variances using respectively the Shapiro-Wilk test and the Fisher test. Therefore, the significance of the mean differences was determined using sided two-sample ttest. All tests were performed using an alpha level of $5 \%$.

\section{Results}

\subsection{Collagen network alignment}

Microstructural observation of the collagen network in mouse dermis during a tensile test showed a progressive alignment of the collagen fibres over all the stretching. It is illustrated in Fig. 2, where we have plotted on the same graph the evolution of the stress versus stretch and the one of the OI, which is a quantitative scalar representing the fraction of collagen fibres aligned in the main direction. The Ol behaviour versus stretch is very similar to the stress behaviour versus stretch, as already reported [21], which indicates that the fibres do not align only in the heel region, where the stress evolves non-linearly, but also - and mostly - in the linear part. This result was obtained for all the WT and transgenic mice alike (see Supplementary Figures 1 and 2).

To get more precise information on the evolution of the fibre orientation with stretch, we directly analysed the evolution of the histograms of orientation, from which the main orientation and the OI were computed.

During the mechanical test, the orientation histogram of collagen fibres evolved from an initial two peak distribution (see the red curve in Fig. 5a) to a single peak distribution centred on the direction of traction (see the blue curve in figure Fig. 5a). Typically, as we imposed stretch on the sample, the 
proportion of fibres oriented in the original directions decreased (this can be seen in Fig. $5 \mathrm{a}$ at $-40^{\circ}$ and $+40^{\circ}$ ), while simultaneously, the proportion of fibres aligned in the direction of traction increased $\left(-10^{\circ}\right.$ and $+10^{\circ}$ in Fig. 5a). The redistribution of fibre orientation first resulted in a one peak distribution with a fairly large plateau (at $\lambda=1.3$ in figure Fig. $5 a$ ), followed by a narrowing of the central peak until the end of the test (from $\lambda=1.4$ in figure Fig. 5a). The fibre orientation histograms of all the samples are displayed in Supplementary Figures 3 and 4.

Figure $5 b$ shows the angle of the maximum of the normalized orientation histogram versus imposed stretch ratio for each step, obtained from SHG images at approximately the same depth for each skin sample. For almost every sample and every depth, the angle of preferred orientation jumped from the angle associated with one of the peaks in initial distribution to a value close to zero, associated with the central peak along the traction direction. This can be associated with a state transition: the whole system shifts from one configuration to another, in a seemingly abrupt fashion. The transition was observed in most images within $5 \%$ strain of the end of the heel region, as in Fig. $5 \mathrm{~b}$. The jump position was almost always the same, although with a small variability between samples and from one image of a SHG stack to the next. The maximal angles versus stretch for all the samples are displayed in Supplementary Figures 5 and 6.

\subsection{Relaxation analysis}

The short time relaxations recorded every 0.05 strain during the pauses in traction (see Fig. 6a) cannot strictly speaking be considered relaxation experiments, as the relaxation time was of the same order of magnitude as the loading time and as the tissue had a different loading history at each stress level. The experiments on longer time relaxations highlight the limitations of fitting data on short time relaxations. For example, to fit the 5-minute relaxations, we needed to introduce a saturation coefficient (i.e. $F_{3}$ ), which corresponds to the fully-relaxed force, while the force decreased to much lower values in the 6-hour relaxations. It is interesting that both short and long relaxations were well fitted by the same type of Prony series (bi-exponential with saturation), albeit with 
different coefficients. This emphasizes that the relaxation times obtained by such fitting approach are strongly dependent of the acquisition time - and so that their comparison should be done carefully. Notably, it implies that short time relaxations are associated with small amplitudes of relaxation, while long time relaxations are associated with large amplitudes of relaxation. It also indicates that we are likely probing only a part of the relaxation mechanics of the skin. Nevertheless, long time relaxation experiments required additional skin samples, as at least one sample is needed per relaxation at a given stretch, so that systematic analysis of the long term relaxation of skin was not possible because of low availability of samples. In contrast, short time relaxation data were obtained from the same experiments as incremental tensile tests, with significant statistical data. As a consequence, the systematic analysis of the short time relaxations and their evolution with stretch offer a simpler insight into the viscous properties of skin, in particular to confront different types of samples and/or loadings.

For a single sample, it was found that all normalized relaxations in the linear part of the stress/stretch curve could be superimposed (beige, orange and red curves in Fig. 6b). On the other hand, the relaxations in the heel region of the stress/stretch curve were always faster, more "efficient", than the relaxations in the linear part (blue and green curves in Fig. 6b).

This was confirmed when analysing the relaxation curves with a bi-exponential fit as described above: the fitting parameters were significantly different between the heel and linear regions for the same type of mice, whether WT or genetically modified (see Fig. 7). Both characteristic times, $t_{1}$ (short) and $t_{2}$ (long), were longer in the linear region than in the heel region. The proportion of force relaxed, in short time relaxation $a_{1}$ and long-time relaxation $a_{2}$, were smaller in the linear region, while the relative constant $s_{0}$ was accordingly larger. Notably, most fitting parameters were not significantly different between WT and transgenic mice, except for the proportion of force relaxed in short time relaxation $\mathrm{a}_{1}$

\subsection{Cycling experiments}


A typical stress/stretch curve is displayed in Fig. 4b. For any given maximal stretch level, the first cycle shows a strong hysteresis, while the following cycle exhibits practically no hysteresis, with both loading and unloading stress curves following closely the previous cycle unloading curve. Interestingly, when further stretching to a higher maximal stretch, the slope of the stress/stretch curve decreases when the stretch first exceeds the values reached in the previous cycle. This decreased slope seems to be the same for all maximal stretches tested $(1.2,1.3$, etc.).

To quantify this hysteresis behaviour, we plotted the stretch ratio $\lambda_{0}$ for which the force first exceeded the sensor's noise level $(0.02 \mathrm{~N})$, against the maximal imposed stretch ratio reached in the previous cycle (see Fig. 8). We observed that the free length of the sample increased mostly after the first cycle at a given maximal stretch ratio: the second cycle at the same maximal stretch ratio had a much lower effect. This was observed at each new series of cycles, at increasing maximal stretches. This is consistent with the effect of limit cycle reported in collagen-rich tissues $[9,13]$, even if increasing the number of cycles may lead to a progressive shift [35].

\subsection{Differences between WT and transgenic mice}

Stress/stretch curves are qualitatively identical for WT and transgenic mice, and we observed the same correspondence between orientation index versus stretch and stress/stretch curves for transgenic and WT mice (see Supplementary Figures 1 and 2). However, as reported in details before [21], statistical differences in the biomechanical properties were observed between WT and transgenic (K14-COL5A1) mice, as summarized in Fig. 9. The tangent modulus was significantly higher for genetically-modified mice, while the heel region was longer, and the slope of the orientation index versus imposed stretch ratio was smaller as in WT mice.

\section{Discussion}

Classical microstructural explanation of the mechanical properties of the skin is based on an unfolding and alignment of the fibres in the heel region, followed by their stretching in the linear region [7]: each fibre brings an elastic linear stress/stretch response as soon as it is aligned in the 
direction of loading. For many years, only qualitative observations were possible $[19,20]$, and they indeed showed more aligned fibres for stretched skin than for resting ones.

Our observation of the OI versus stretch evolution indicates that the fibres align to the direction of traction continuously during the whole stretching (see Fig. 2). As our results were in contradiction with the classical interpretation, we developped a new interpretation in order to explain all our observations simultaneously.

The evolution of the OI with stretch and its proportionality with stress has two main implications.

Firstly, with elastic collagen fibres aligning progressively as evidenced by the continuous increase in OI (see Fig. 2), the constant addition of elastic contributions of the fibres would make the linear part of the stress/stretch curve non-linear (more than linear, in fact). It then becomes clear that the collagen fibres cannot assume an elastic behaviour. It suggests that they should instead add a certain amount of stress to the tissue, at any given stretch ratio.

Secondly, little reorganization was measured in the toe and heel regions of the stress/stretch curve: the OI barely increased (see Fig. 2). It suggests that the rearrangement of the fibre network is not the the main mechanism implicated in that part of the stress/stretch curve.

A good hypothesis for the predominant microstructural mechanism in the linear part would be that the collagen fibres have a plastic behavior, which could be caused by inner sliding. Recent observations of sliding between collagen fibrils in tendons suggest a model of sliding proteoglycan bonds within the fibres $[36,37]$. The strain is then distributed between the collagen fibrils and a proteoglycan-rich matrix, which is mainly sheared. The increase of the free length with repeated cycling reported in the previous section (see Fig. 8) is in good agreement with this interpretation: as sliding occurs, the skin undergoes a plastic elongation.

The mechanism of inner sliding resulting in plastic fibres could explain the linear region of the stress/stretch curve. However, this mechanism does not account for the non-linear stiffening of skin in the heel region. This phenomenon could be an inherent effect of the organization of collagen fibres in a 2D network: in the toe and heel regions, the fibres perpendicular to the direction of 
traction would need to buckle and bend before reorientation can occur. Recent numerical results [38-40] support this interpretation. These studies were inspired by experiments on low density collagen gels [41] and simulated large network of elastic collagen fibres connected by flexible junctions. However, these studies did not generally introduce the non-fibrillar matrix surrounding the fibres, which may have a strong influence in the non-linear response [42], especially since it is modifying the buckling properties.

The question of the role of the viscosity in the response of the system is also of primary importance. Indeed, the evolution of the histogram of fibre orientation (see Fig. 5) seems to indicate that modelling the non-fibrillar matrix as an elastic affine material, as commonly found in the literature $[2,13,15,16]$, is not adequate. Then the fibres would only be driven by the deformation of the bulk material they are embedded in. The histogram of fibre orientation in a deformed state would then merely be a narrowed version of the initial distribution. To actually modify the shape of the fibre orientation histogram, the fibres need to be embedded in a viscous environment. Structural effects are also possible, but seem less likely for long fibres as collagen ones [43].

Yet, if the heel and toe regions are indeed dominated by buckling of fibres perpendicular to traction and bending of reticulation points, while the linear region sees mainly the collagen fibres undergoing inner sliding, this would imply a shift in the predominant microstructural mechanism between heel and linear regions. The change in the relaxation behaviour observed (see Fig. 6 and 7), as well as the state transition evidenced by the orientation histograms (see Fig. 5b), can be clues to that shift in predominant mechanism.

These two mechanisms, each at a different length scale, are summarized in Fig. 10. Inside the fibres, at the scale of the fibril to fibril interface, viscoelastic proteoglycan bonds could explain why the linear part of the stress/stretch curve remains linear while the orientation index increases continuously. At the scale of the collagenous network, a bending dominated elasticity could explain simultaneously the non-linear stiffening and the J-shaped curve of the orientation index against imposed strain curve. 
The results observed in cyclic experiments are well explained by our model. The plastic response of the fibres aligned in the direction of traction will lead to an increase of the resting length of the network when first loading the sample. The elastic response of the fibres and the relaxation of the network could explain the fast decrease of the force observed when unloading the sample. The force cannot become negative since the dermis is a membrane: it buckles at very low forces. As subsequent loadings in the same range of macroscopic stretches do not modify the structure of the network nor the length of the fibres, the stress/stretch curves superimpose. However, larger stretches will load again the fibres in their plastic regime, leading to a further increase of the resting length and again to a large hysteresis loop. A change in the slope of the stretch/stress curve is then expected: for stretches that were already reached in the previous loading history, the slope is associated with the elastic response of the fibres and network, while beyond this point the slope is dominated by the plastic response.

Our model also fits well with the results on transgenic mice skin (see Fig. 9), with the addition of supplementary non-fibrillar matrix as an important player in the microscopic biomechanical behavior of skin. In the transgenic mice considered (K14-COL5A1), there is an addition of small collagen $\mathrm{V}$ homotypic fibrils, presenting a diameter smaller than $10 \mathrm{~nm}$, mainly at the junction between the epidermis and the dermis, and diffusing in the dermis [32]. These homotypic fibrils are too small to be observed in SHG images (10nm diameter, less than the minimal visible diameter: $30 \mathrm{~nm}$ [34]), and thus contribute to the surrounding environment of the "classical" heterotypic collagen fibres. All other elements of the dermis are believed to remain identical to WT mice. The addition of these small collagen $V$ fibrils could intervene in each of the mechanisms we suggested, summarized in Fig. 10.

At the scale of the heterotypic fibre, the small collagen $V$ homotypic fibrils may slow down the mechanics of inner sliding between fibrils both by steric effect (surrounding the fibre or penetrating inside the fibre, between fibrils) and by increasing local shears: the heterotypic collagen fibres could then withstand the deformation more efficiently. Since the heterotypic fibre density was found to be 
normal in transgenic mice (as judged by histology and TEM in [32]), an increased mechanical contribution of each individual fibre would result in an increase of the stress in the whole tissue. This is consistent with the higher tangent modulus observed for transgenic mice. Notably, the mechanism by which the collagen $V$ fibrils could interfere with the sliding between fibrils, inside each heterotypic collagen fibre, is yet to be determined. In particular, it has to be noted that no insertion of collagen $\mathrm{V}$ homotypic fibrils inside heterotypic collagen fibres has been observed with TEM images. It is possible that the collagen $V$ fibrils impede fibril-fibril sliding either by bonding with different heterotypic fibrils through multiple crosslinks, and/or by hampering the flow of other non fibrillar elements through steric effect.

At the scale of the non-fibrillar matrix, the presence of collagen $V$ fibrils is expected to increase either the stiffness or the viscosity, which could impede the heterotypic collagen fibres' movements. The fibres perpendicular to the traction direction could then resist buckling more easily, because the collagen $\vee$ fibrils would act like props for heterotypic fibres. This could be an explanation for the lengthened heel region in transgenic mice.

Finally, we observed that the heterotypic fibres align continuously in the direction of traction during the linear region of the stress/stretch curve, causing the stress to rise (see Fig. 2). Yet, if the heterotypic fibres movements are obstructed by collagen $V$ fibrils, the reorganization would be more difficult. This would lead to a decreased slope of the orientation index against stretch curve, as observed.

\section{Conclusion}

The complementary set of experiments carried out demonstrates that the classical model, in which the linear part of the J-shaped stress/stretch curve is attributed to the extension of aligned collagen fibres, is not appropriate for skin, although adequate for tendon-like connective tissues. In contrast, our multiscale experiments show that the fraction of fibres aligned in the direction of traction increases non-linearly with stretch and follows exactly the stress/stretch curve throughout the tensile test. Thus, contrary to a classical claim in biomechanics publications, the collagen fibres do 
not behave elastically within the dermis structure. Instead, we propose that the collagen fibres have a plastic behaviour, each fibre contributing by a fixed quantity to the total force.

All the data obtained from various loadings are combined to propose new mechanisms coupling the scale of the individual fibres, and the scale of the full network. The heel region is associated to a structural response of the network of fibres, and in particular the buckling of transverse fibres. The linear part is interpreted as a progressive alignment of the network of fibres, each fibre being able to provide only a given stress due to inter-fibrils sliding inside each fibre.

This approach has been used to explain the effect of a simple modification of the dermis microstructure in genetically-modified mice. This new interpretation emphasizes the importance of the non-fibrillar matrix, in determining the mechanical behaviour of the skin, its viscosity and its capability to stabilize the fibres network appearing as key elements to understand the relation between the mechanical response and the remodelling of the collagen matrix in the dermis, and presumably in other complex connective tissues.

\section{Acknowledgement}

We thank Xavier Solinas, Jean-Marc Sintès and Vincent de Greef for technical implementation of the setups, Nadine Aguilera for breeding mice and maintaining transgenic strains (PBES, SFR BiosciencesGerland, Lyon, France) and Isabelle Lamarre-Jouenne for technical support in skin handling. This work was supported by grants from Ecole Polytechnique (interdisciplinary project), from Agence Nationale de la Recherche (ANR-13-BS09-0004-02 and ANR-10-INBS-04).

\section{Disclosures}

We have no competing interest.

\section{References}

[1] G.L. Wilkes, I.A. Brown, R.H. Wildnauer, The biomechanical properties of skin, Crit. Rev. Biomed. Eng. 1 (1973) 453-495.

[2] R.B. Groves, S.A. Coulman, J.C. Birchall, S.L. Evans, An anisotropic, hyperelastic model for skin: Experimental measurements, finite element modelling and identification of parameters for human and murine skin, J. Mech. Behav. Biomed. Mater. 18 (2013) 167-180. 
[3] C. Bismuth, C. Gerin, E. Viguier, D. Fau, F. Dupasquier, L. Cavetier, L. David, C. Carozzo, The biomechanical properties of canine skin measured in situ by uniaxial extension, J. Biomech. 47 (2014) 1067-1073.

[4] F. Xu, T.J. Lu, K.A. Seffen, Biothermomechanical behavior of skin tissue, Acta Mech. Sin. 24 (2008) 1-23.

[5] M.F. Leyva-Mendivil, A. Page, N.W. Bressloff, G. Limbert, A mechanistic insight into the mechanical role of the stratum corneum during stretching and compression of the skin, J. Mech. Behav. Biomed. Mater. 49 (2015) 197-219.

[6] M. Geerligs, Skin layer mechanics, T.U. Eindhoven, 2010.

[7] D.R. Veronda, R.A. Westmann, Mechanical characterization of skin-finite deformations, J. Biomech. 3 (1970) 111-124.

[8] D.L. Bader, P. Bowker, Mechanical characteristics of skin and underlying tissues in vivo, Biomaterials. 4 (1983) 305-308.

[9] Y. Goulam Houssen, I. Gusachenko, M.-C. Schanne-Klein, J.-M. Allain, Monitoring micrometerscale collagen organization in rat-tail tendon upon mechanical strain using second harmonic microscopy, J. Biomech. 44 (2011) 2047-2052.

[10] A. Benoit, G. Latour, S.-K. Marie-Claire, J.-M. Allain, Simultaneous microstructural and mechanical characterization of human corneas at increasing pressure, J. Mech. Behav. Biomed. Mater. (2015).

[11] Y.C. Fung, Biomechanics. Mechanical Properties of Living Tissues, second edition, SpringerVerlag, 1993.

[12] M. Ottenio, D. Tran, A. Ní Annaidh, M.D. Gilchrist, K. Bruyère, Strain rate and anisotropy effects on the tensile failure characteristics of human skin, J. Mech. Behav. Biomed. Mater. 41 (2015) 241-250.

[13] Y. Lanir, O. Lokshin, Viscoelasticity and Preconditioning of Rat Skin Under Uniaxial Stretch: Microstructural Constitutive Characterization, J. Biomech. Eng. 131 (2009) 31009.

[14] A.S. Caro-Bretelle, P.N. Gountsop, P. lenny, R. Leger, S. Corn, I. Bazin, F. Bretelle, Effect of sample preservation on stress softening and permanent set of porcine skin, J. Biomech. 48 (2015) 3135-3141.

[15] J.W.Y. Jor, M.P. Nash, P.M.F. Nielsen, P.J. Hunter, Estimating material parameters of a structurally based constitutive relation for skin mechanics, Biomech. Model. Mechanobiol. 10 (2010) 767-778.

[16] T.K. Tonge, L.M. Voo, T.D. Nguyen, Full-field bulge test for planar anisotropic tissues: Part II - A thin shell method for determining material parameters and comparison of two distributed fiber modeling approaches, Acta Biomater. 9 (2013) 5926-5942.

[17] G.A. Holzapfel, M. Stadler, C.A.J. Schulze-Bauer, A Layer-Specific Three-Dimensional Model for the Simulation of Balloon Angioplasty using Magnetic Resonance Imaging and Mechanical Testing, Ann. Biomed. Eng. 30 (2002) 753-767.

[18] A. Pandolfi, F. Manganiello, A model for the human cornea: constitutive formulation and numerical analysis, Biomech. Model. Mechanobiol. 5 (2006) 237-246.

[19] I.A. Brown, Scanning Electron-microscope Study of Effects of Uniaxial Tension On Human Skin, Br. J. Dermatol. 89 (1973) 383-393.

[20] S.M. Belkoff, R.C. Haut, A structural model used to evaluate the changing microstructure of maturing rat skin, J. Biomech. 24 (1991) 711-720.

[21] S. Bancelin, B. Lynch, C. Bonod-Bidaud, G. Ducourthial, S. Psilodimitrakopoulos, P. Dokládal, J.M. Allain, M.-C. Schanne-Klein, F. Ruggiero, Ex vivo multiscale quantitation of skin biomechanics in wild-type and genetically-modified mice using multiphoton microscopy, Sci. Rep. 5 (2015) 17635.

[22] J.T. Keyes, D.G. Haskett, U. Utzinger, M. Azhar, J.P.V. Geest, Adaptation of a planar microbiaxial optomechanical device for the tubular biaxial microstructural and macroscopic characterization of small vascular tissues, J. Biomech. Eng. 133 (2011) 75001. 
[23] P.J. Campagnola, A.C. Millard, M. Terasaki, P.E. Hoppe, C.J. Malone, W.A. Mohler, ThreeDimensional High-Resolution Second-Harmonic Generation Imaging of Endogenous Structural Proteins in Biological Tissues, Biophys J. 82 (2002) 493-508.

[24] W.R. Zipfel, R.M. Williams, W.W. Webb, Nonlinear magic: multiphoton microscopy in the biosciences, Nat. Biotechnol. 21 (2003) 1369-1377.

[25] M. Strupler, A.-M. Pena, M. Hernest, P.-L. Tharaux, J.-L. Martin, E. Beaurepaire, M.-C. SchanneKlein, Second harmonic imaging and scoring of collagen in fibrotic tissues, Opt Express. 15 (2007) 4054-4065.

[26] I. Gusachenko, V. Tran, Y.G. Houssen, J.-M. Allain, M.-C. Schanne-Klein, Polarization-Resolved Second-Harmonic Generation in Tendon upon Mechanical Stretching, Biophys. J. 102 (2012) 2220-2229.

[27] S. Nesbitt, W. Scott, J. Macione, S. Kotha, Collagen Fibrils in Skin Orient in the Direction of Applied Uniaxial Load in Proportion to Stress while Exhibiting Differential Strains around Hair Follicles, Materials. 8 (2015) 1841-1857.

[28] J.T. Keyes, D.R. Lockwood, B.R. Simon, J.P. Vande Geest, Deformationally dependent fluid transport properties of porcine coronary arteries based on location in the coronary vasculature, J. Mech. Behav. Biomed. Mater. 17 (2013) 296-306.

[29] A. Mauri, M. Perrini, J.M. Mateos, C. Maake, N. Ochsenbein-Koelble, R. Zimmermann, M. Ehrbar, E. Mazza, Second harmonic generation microscopy of fetal membranes under deformation: Normal and altered morphology, Placenta. 34 (2013) 1020-1026.

[30] A. Mauri, A.E. Ehret, M. Perrini, C. Maake, N. Ochsenbein-Kölble, M. Ehrbar, M.L. Oyen, E. Mazza, Deformation mechanisms of Human amnion: Quantitative studies based on second harmonic generation microscopy, J. Biomech. (2015).

[31] C. Jayyosi, M. Coret, K. Bruyère-Garnier, Characterizing liver capsule microstructure via in situ bulge test coupled with multiphoton imaging, J. Mech. Behav. Biomed. Mater. 54 (2016) 229243.

[32] C. Bonod-Bidaud, M. Roulet, U. Hansen, A. Elsheikh, M. Malbouyres, S. Ricard-Blum, C. Faye, E. Vaganay, P. Rousselle, F. Ruggiero, In Vivo Evidence for a Bridging Role of a Collagen V Subtype at the Epidermis-Dermis Interface, J. Invest. Dermatol. 132 (2012) 1841-1849.

[33] K. Konig, I. Riemann, High-resolution multiphoton tomography of human skin with subcellular spatial resolution and picosecond time resolution, J Biomed Opt. 8 (2003) 432-439.

[34] S. Bancelin, C. Aimé, I. Gusachenko, L. Kowalczuk, G. Latour, T. Coradin, M.-C. Schanne-Klein, Determination of collagen fibril size via absolute measurements of second-harmonic generation signals, Nat Commun. 5 (2014).

[35] M.-O. Kiss, N. Hagemeister, A. Levasseur, J. Fernandes, B. Lussier, Y. Petit, A low-cost thermoelectrically cooled tissue clamp for in vitro cyclic loading and load-to-failure testing of muscles and tendons, Med. Eng. Phys. 31 (2009) 1182-1186.

[36] R. Puxkandl, I. Zizak, O. Paris, J. Keckes, W. Tesch, S. Bernstorff, P. Purslow, P. Fratzl, Viscoelastic properties of collagen: synchrotron radiation investigations and structural model, Philos. Trans. R. Soc. B Biol. Sci. 357 (2002) 191-197.

[37] H.R. Screen, D.L. Bader, D.A. Lee, J.C. Shelton, Local strain measurement within tendon, Strain. 40 (2004) 157-163.

[38] A.M. Stein, D.A. Vader, D.A. Weitz, L.M. Sander, The micromechanics of three-dimensional collagen-I gels, Complexity. 16 (2011) 22-28.

[39] A.J. Licup, A. Sharma, F.C. MacKintosh, Elastic regimes of subisostatic athermal fiber networks, Phys. Rev. E. 93 (2016).

[40] A. Mauri, R. Hopf, A.E. Ehret, C.R. Picu, E. Mazza, A discrete network model to represent the deformation behavior of human amnion, J. Mech. Behav. Biomed. Mater. 58 (2016) 45-56.

[41] D. Vader, A. Kabla, D. Weitz, L. Mahadevan, Strain-Induced Alignment in Collagen Gels, PLoS ONE. 4 (2009) e5902.

[42] L. Zhang, S.P. Lake, V.H. Barocas, M.S. Shephard, R.C. Picu, Cross-linked fiber network embedded in an elastic matrix, Soft Matter. 9 (2013) 6398. 
[43] W. Krasny, C. Morin, S. Avril, H. Magoariec, C. Hellmich, The concept of frozen elastic energy as a consequence of changes in microstructure morphology, Comput. Methods Biomech. Biomed. Engin. 18 (2015) 1966-1967.

\section{Figures captions}

Figure 1: Schematic representation of the experimental set-up.

(a) Under the multiphoton microscope. Hydration is ensured with an immersion gel. A typical image

in rest state obtained with multiphoton microscope is shown. (b) Outside the multiphoton microscope. Hydration is ensured by regularly spraying of the sample with mineral water. A white light source lights up the sample, and images are taken every second using a digital camera. A typical image in rest state obtained with the digital camera is displayed, showing the pattern of Indian ink dots.

Figure 2: Typical nominal stress/stretch curve (black) and corresponding Ol/stretch curve (red) for a tensile test on WT mice skin performed under the multiphoton microscope. The collagen alignment follows exactly the stress. The red arrows indicate the pauses in the traction for multiphoton imaging.

Figure 3: Relaxation analysis.

(a) Short relaxation (6 minutes) obtained during a pause in the traction for multiphoton imaging. (b) Long relaxation (6 hours) obtained during a relaxation experiment outside the microscope.

Figure 4: Cycling experiments outside the multiphoton microscope.

(a) Cyclic loading path: two complete cycles were imposed with a maximum imposed stretch ratio of $\lambda=1.05$, then two cycles at $\lambda=1.1$, two cycles at $\lambda=1.2$, two cycles at $\lambda=1.3$, and then finally stretched the sample up to rupture. (b) Corresponding nominal stress/stretch curve.

Figure 5: Evolution of collagen network organization with imposed stretch ratio.

(a) Histogram of fibre orientation over $10 \mu \mathrm{m}$ thickness throughout the mechanical test, from $\lambda=1$ to $\lambda=1.6(\lambda=1.05,1.15 \ldots$ are not shown for the sake of clarity). (b) Stress/stretch curve (black) and 
maximum of the normalized histogram of fibre orientation for a SHG image at a given depth (red), against the imposed stretch ratio, for the same sample.

Figure 6: Comparison of relaxation behaviour in heel and linear regions for a WT mouse sample.

(a) Nominal stress/stretch curve. (b) Corresponding normalized relaxations in the heel (blue and green) and linear (beige, orange, red) parts. All normalized force/time curves were processed with a moving average using Matlab (number of points=9).

Figure 7: Comparison of relaxation behaviour in heel and linear regions.

(a) Short time $t_{1}$, (b) long time $t_{2}$, (c) amplitude for the short time relaxation $a_{1}$, (d) amplitude for the long time relaxation $\mathrm{a}_{2}$, (e) relative constant for the relaxation $\mathrm{s}_{0}$. Error bars correspond to SEM. * stands for $p<0.05, * *$ for $p<0.01$ and ${ }^{* * *}$ for $p<0.001$.

Figure 8: Stretch ratio $\lambda_{0}$ for which the force first exceeded the sensor's noise level during the stretching part of a cycle against the maximum imposed stretch ratio of the previous cycle, for the same sample as in Fig. 3. The colour of a dot is the one of the previous cycle on the loading path in Fig. 3.

Figure 9: Comparison of WT and K14-COL5A1 mice biomechanical behaviour.

(a) Tangent modulus, (b) heel region length, (c) slope of OI variation. Error bars corresponds to SEM. * stands for $p<0.05, * *$ for $p<0.01$ and $* * *$ for $p<0.001$. Adapted from [34].

Figure 10: Schematic representation of the three microstructural mechanisms proposed for our interpretation: 1- at the scale of the fibre, sliding between fibrils connected by proteoglycan bonds, 2- at the scale of the collagenous network, bending of reticulation points and fibre buckling, 3-finally, the mechanical properties of the non-fibrillar matrix contribute to the general mechanical behaviour of skin. The left part of the figure indicates in which region of the stress/stretch curve each mechanism is important. 